\title{
Risk assessment in case of toxic chemical emission at railway transport
}

\author{
Viktoriia Biliaieva ${ }^{1, *}$, Polina Mashykhina ${ }^{2}$, Ivan Kalashnikov ${ }^{3}$, Oleksandr Berlov ${ }^{4}$, and Ivan Kravets $^{5}$ \\ ${ }^{1}$ DNU, Department aerohydromechanics and energy and masstransfer, 49050 Dnipro, Kozakov Street, 18, Ukraine \\ ${ }^{2}$ DNURT, Department hydraulics and water supply, 49010 Dnipro, Lazaryan Street, 2, Ukraine \\ ${ }^{3}$ Kharkiv Branch Office «Design and Research Institute of Railway Transport» of the PJS «Ukrainian Railway», 61052 Kharkiv, \\ Kotliar Street, 7, Ukraine \\ ${ }^{4}$ PSACEA, 49600 Dnipro, Chernyshevsky Street, 24a, Ukraine \\ ${ }^{5}$ DNURT, Lviv branch, 79052 Lviv, I. Blazhkevich Street, 12a, Ukraine
}

\begin{abstract}
Risk assessment during emission of toxic chemicals at railway transport is the problem of great scientific interest. To make such assessment we need special computer models. At present, in Ukraine,we have lack of such models. The authors present numerical models for territorial risk assessment in case of organized emissionsat railway transport (for example, emissions during locomotive movement) and in case of accident emissions (accident spills of dangerous cargo, emissions of $\mathrm{NH}_{3}$ from railway tank, etc.). The basis of the developed numerical models is the system of fundamental equations of fluid dynamics. These equations are solved numerically using implicit schemes of splitting. The developed models allow to take into account some important factors which influence the territorial risk value: probability of atmosphere conditions, train route, transport infrastructure at railway stations, probability of emission site.Also the process of pollutant chemical transformation in the atmosphere is taken into account in the developed models. The developed models allow to predict territorial risk in case of moving source of emission (moving damaged railway tank). The results of numerical experiments are presented. These results illustrate territorial risk maps for different sites near Prydniprovska railway.
\end{abstract}

\section{Relevance}

During the operation of rail transport emissions of pollutants into the atmosphere can be divided into two classes. The first class is organized emissions to the atmosphere during the movement of diesel locomotives or other technical means. The second class of emissions is accidental emissions during transportation of dangerous cargo. In the event of an accident during the transportation of chemically hazardous substances, there is a risk of large-scale environmental pollution and damage to people. When considering both problems, a problem arises in assessing the territorial risk of toxic damage to humans $[1-4,13,15-20]$. To carry out such assessment we need special mathematical models $[5,6$, $7,9,, 10,12]$. At present, such models are absent in Ukraine. Therefore, there is an important task of creating effective methods for assessing the territorial risk of various emissions of chemically hazardous substances in railway transport.

\section{Governing equations}

To simulate the process of toxic chemical dispersion in the atmosphere the transport equation is used $[7,8,12$, 14]:

$$
\begin{gathered}
\frac{\partial C}{\partial t}+\frac{\partial u C}{\partial x}+\frac{\partial v C}{\partial y}+\frac{\partial w C}{\partial z}+\sigma C= \\
\frac{\partial}{\partial x}\left(\mu_{x} \frac{\partial C}{\partial x}\right)+\frac{\partial}{\partial y}\left(\mu_{y} \frac{\partial C}{\partial y}\right)+\frac{\partial}{\partial z}\left(\mu_{z} \frac{\partial C}{\partial z}\right)+ \\
+\sum Q_{i}(t) \delta\left(x-x_{i}\right) \delta\left(y-y_{i}\right) \delta\left(z-z_{i}\right),
\end{gathered}
$$

where $u, v, w$ are the velocity components in $x, y$ and $z$ direction respectively; $C$ is the concentration of toxic chemical; $\sigma$ is the parameter taking into account the process of toxic gas decay or rain wash out; $\mu_{x}, \mu_{y}, \mu_{z}$ are the coefficients of turbulent diffusion in $x, y$ and $z$ direction respectively; $x_{i}, y_{i}, z_{i}$ are the coordinates of point source of emission; $Q_{i}(t)$ is the intensity of pollutant emission; $\delta\left(x-x_{\mathrm{i}}(\mathrm{t})\right) \delta\left(y-y_{\mathrm{i}}(\mathrm{t})\right) \delta\left(z-z_{\mathrm{i}}\right)$ is Dirac delta-function.

In the developed numerical model, the following profile of velocity component $u$ and coefficient of diffusion $\mu_{z}$ is used [5]:

$$
u=u_{1}\left(\frac{z}{z_{1}}\right)^{n}, \mu_{z}=k_{1}\left(\frac{z}{z_{1}}\right)^{m}
$$


where $u_{1}$ is the velocity at height $z_{1} ; k_{l}=0,2 ; n=0,16$; $m \approx 1$. The following models to calculate the other diffusive coefficients are used:

$$
\mu_{y}=\mu_{x}, \mu_{y}=k_{0} u,
$$

where $k_{0}$ is the empirical parameter.

The transport equation is used with the following boundary conditions $[1,5]$ :

- inlet boundary: $\left.\mathrm{C}\right|_{\text {inlet }}=C_{E}$, where $C_{E}$ is the known concentration (very often $C_{E}=0$ );

- outlet boundary: in numerical model the condition $C(i+1, j, k)=C(i, j, k)$ is used (this boundary condition means that we neglect the process of diffusion at this plane);

- top boundary and ground surface $\partial \mathrm{C} / \partial \mathrm{n}=0$.

To develop the numerical model the implicit difference scheme was used.

\section{Modeling of land surface contamination}

In case of accidents during transportation of toxic chemicals, an important problem is arisen: it is necessary justify economic losses, caused by land contamination. Worthy of note that is practically impossible to measure contamination area very quickly after an accident, because of lack of special equipment, big areas, which were affected by pollutant emission. So, the mathematical modeling is the main instrument to calculate the losses after the accident.

The following dependence is used to calculate mass of pollutant which is "captured" by land surface:

$$
G=\int_{0}^{T} d t \iint_{S, z=0}\left(w_{s}+\mu \alpha\right) C(x, y, z=0, t) d s,
$$

where $S$ - specific area of the surface of the region (forest, crops, etc.), $t$ - current time; $\alpha>0$ - coefficient taking into account the "capture" of part of the impurity by the earth's surface [8]; $T$ is the period of integration.

For calculation of this integral the formula of rectangles is used.

Worthy of note, that to calculate parameter $\mathrm{G}$, it is necessary to know concentration field near the land surface. Besides, concentration near the land surface is changed during time. This concentration can be computed using equation (1).

\section{Numerical integration}

For numerical integration of the equation (1) finitedifference methods are used. First, equation (1) was split into a sequence of next equations:

$$
\frac{\partial C}{\partial t}+\frac{\partial u C}{\partial x}=\frac{\partial}{\partial x}\left(\mu_{x} \frac{\partial C}{\partial x}\right), \frac{\partial C}{\partial t}+\frac{\partial v C}{\partial y}=\frac{\partial}{\partial y}\left(\mu_{y} \frac{\partial C}{\partial y}\right),
$$

$$
\begin{gathered}
\frac{\partial C}{\partial t}+\frac{\partial\left(w-w_{g}\right) C}{\partial z}=\frac{\partial}{\partial z}\left(\mu_{z} \frac{\partial C}{\partial z}\right), \\
\frac{\partial C}{\partial t}+\sigma C=\sum Q_{i}(t) \delta\left(x-x_{i}(t)\right) \delta\left(y-y_{i}(t)\right) .
\end{gathered}
$$

Each of first three equations from system (3) describes hazardous substances movement in one particular direction. This splitting is the bases for developing of locally-unidimensional difference scheme. For numerical integration of the first three equations, implicit alternately triangular difference scheme is used.

To build difference scheme, first of all the convective derivatives are represented as following [12]:

$$
\begin{gathered}
\frac{\partial u C}{\partial x}=\frac{\partial u^{+} C}{\partial x}+\frac{\partial u^{-} C}{\partial x}, \frac{\partial v C}{\partial y}=\frac{\partial v^{+} C}{\partial y}+\frac{\partial v^{-} C}{\partial y}, \\
\frac{\partial w C}{\partial z}=\frac{\partial w^{+} C}{\partial z}+\frac{\partial w^{-} C}{\partial z},
\end{gathered}
$$

Where

$$
\begin{gathered}
u^{+}=\frac{u+|u|}{2} ; u^{-}=\frac{u-|u|}{2} ; v^{+}=\frac{v+|v|}{2} ; v^{-}=\frac{v-|v|}{2} ; \\
w^{+}=\frac{w+|w|}{2} ; w^{-}=\frac{w-|w|}{2} .
\end{gathered}
$$

After that, approximation of the second derivatives is made by formulas from [12]:

$$
\begin{aligned}
& \frac{\partial}{\partial x}\left(\mu_{x} \frac{\partial C}{\partial x}\right) \approx \mu_{x} \frac{C_{i+1, j, k}^{n+1}-C_{i, j, k}^{n+1}}{\Delta x^{2}}-\mu_{x} \frac{C_{i, j, k}^{n+1}-C_{i-1, j, k}^{n+1}}{\Delta x^{2}}= \\
& =M_{x x}^{-} C^{n+1}+M_{x x}^{+} C^{n+1}, \\
& \frac{\partial}{\partial y}\left(\mu_{y} \frac{\partial C}{\partial y}\right) \approx \mu_{y} \frac{C_{i, j+1, k}^{n+1}-C_{i, j, k}^{n+1}}{\Delta y^{2}}-\mu_{y} \frac{C_{i, j, k}^{n+1}-C_{i, j-1, k}^{n+1}}{\Delta y^{2}}= \\
& =M_{y y}^{-} C^{n+1}+M_{y y}^{+} C^{n+1}, \\
& \frac{\partial}{\partial z}\left(\mu_{z} \frac{\partial C}{\partial z}\right) \approx \mu_{z} \frac{C_{i, j, k+1}^{n+1}-C_{i, j, k}^{n+1}}{\Delta z^{2}}-\mu_{z} \frac{C_{i, j, k}^{n+1}-C_{i, j, k-1}^{n+1}}{\Delta z^{2}}= \\
& =M_{z z}^{-} C^{n+1}+M_{z z}^{+} C^{n+1} .
\end{aligned}
$$

For approximation of the convective derivatives formulas from [12] are used:

$$
\begin{aligned}
& \frac{\partial u^{+} C}{\partial x} \approx \frac{u_{i+1, j, k}^{+} C_{i, j, k}^{n+1}-u_{i, j, k}^{+} C_{i-1, j, k}^{n+1}}{\Delta x}=L_{x}^{+} C^{n+1}, \\
& \frac{\partial u^{-} C}{\partial x} \approx \frac{u_{i+1, j, k}^{-} C_{i+1, j, k}^{n+1}-u_{i, j, k}^{-} C_{i, j, k}^{n+1}}{\Delta x}=L_{x}^{-} C^{n+1}, \\
& \frac{\partial v^{+} C}{\partial y} \approx \frac{v_{i, j+1, k}^{+} C_{i, j, k}-v_{i, j, k}^{+} C_{i, j-1, k}}{\Delta y}=L_{y}^{+} C^{n+1} \\
& \frac{\partial v^{-} C}{\partial y} \approx \frac{v_{i, j+1, k}^{-} C_{i, j+1, k}-v_{i, j, k}^{-} C_{i, j, k}}{\Delta y}=L_{y}^{-} C^{n+1}
\end{aligned}
$$




$$
\begin{aligned}
& \frac{\partial w^{+} C}{\partial z} \approx \frac{w_{i, j, k+1}^{+} C_{i, j, k}-w_{i, j, k}^{+} C_{i, j, k-1}}{\Delta z}=L_{z}^{+} C^{n+1} \\
& \frac{\partial w^{-} C}{\partial z} \approx \frac{w_{i, j, k+1}^{-} C_{i, j, k+1}-w_{i, j, k}^{-} C_{i, j, k}}{\Delta z}=L_{z}^{-} C^{n+1}
\end{aligned}
$$

Difference scheme for calculation chemically hazardous substance concentration is considered on the example of the first equation from system (3) because of similarity of the second and the third to the first one. Difference scheme is written as follow:

- on the first step of splitting:

$$
\frac{C_{i, j, k}^{k}-C_{i, j, k}^{n}}{\Delta t}+L_{x}^{+} C^{k}=M_{x x}^{+} C^{k}+M_{x x}^{-} C^{n}
$$

- on the second step of splitting:

$$
\frac{C_{i, j, k}^{n+1}-C_{i, j, k}^{k}}{\Delta t}+L_{x}^{-} C^{n+1}=M_{x x}^{+} C^{n}+M_{x x}^{-} C^{n+1} .
$$

Difference scheme has the similar form for the second and the third equations from (3).

Unknown value of concentration $\mathrm{C}$ at each step of splitting (4), (5) is calculated with explicit formula of «running calculation». The last equation from (3) is solved with using of Euler method.

Further, the physical splitting is carried out on the differential level. When doing this splitting, the processes of convection, diffusion, «work» of sources and runoffs need to be separated. Splitting has following appearance [8]:

$$
\begin{gathered}
\frac{\partial C}{\partial t}+\frac{\partial u C}{\partial x}+\frac{\partial v C}{\partial y}+\frac{\partial\left(w-w_{S}\right) C}{\partial z}=0 \\
\frac{\partial C}{\partial t}=\frac{\partial}{\partial x}\left(\mu_{x} \frac{\partial C}{\partial x}\right)+\frac{\partial}{\partial y}\left(\mu_{y} \frac{\partial C}{\partial y}\right)+\frac{\partial}{\partial z}\left(\mu_{z} \frac{\partial C}{\partial z}\right) \\
\frac{\partial C}{\partial t}=\sum Q_{i}(t) \delta\left(x-x_{i}(t)\right) \delta\left(y-y_{i}(t)\right) \delta\left(z-z_{i}\right) . \\
\frac{\partial C}{\partial t}+\sigma C=0
\end{gathered}
$$

Need to be noted, that the third equation from (6) defines changing of hazardous substance concentration under the action of emission source with rate $\mathrm{Q}$.

Equation (7) defines changing of hazardous substance concentration in the particular point under the chemical decay.

It is known, that to perform computational experiments other methods of numerical integration to control the correctness of numerical calculations need to be used. That is why developing of the other difference scheme for numerical integration of equation (1) is considered.

To describe this difference scheme, following dependencies are used [12]:

$$
\begin{gathered}
\frac{\partial C}{\partial t} \approx \frac{C_{i j}^{n+1}-C_{i j}^{n}}{\Delta t}, \frac{\partial u C}{\partial x}=\frac{\partial u^{+} C}{\partial x}+\frac{\partial u^{-} C}{\partial x}, \\
\frac{\partial v C}{\partial y}=\frac{\partial v^{+} C}{\partial y}+\frac{\partial v^{-} C}{\partial y}, \frac{\partial w C}{\partial z}=\frac{\partial w^{+} C}{\partial z}+\frac{\partial w^{-} C}{\partial z}
\end{gathered}
$$

where

$$
\begin{aligned}
& u^{+}=\frac{u+|u|}{2} ; u^{-}=\frac{u-|u|}{2} ; v^{+}=\frac{v+|v|}{2} ; v^{-}=\frac{v-|v|}{2} ; \\
& w^{+}=\frac{w+|w|}{2} ; w^{-}=\frac{w-|w|}{2} \text {. } \\
& \frac{\partial}{\partial x}\left(\mu_{x} \frac{\partial C}{\partial x}\right) \approx \mu_{x} \frac{C_{i+1, j, k}^{n+1}-C_{i, j, k}^{n+1}}{\Delta x^{2}}-\mu_{x} \frac{C_{i, j, k}^{n+1}-C_{i-1, j, k}^{n+1}}{\Delta x^{2}}= \\
& =M_{x x}^{-} C^{n+1}+M_{x x}^{+} C^{n+1}, \\
& \frac{\partial}{\partial y}\left(\mu_{y} \frac{\partial C}{\partial y}\right) \approx \mu_{y} \frac{C_{i, j+1, k}^{n+1}-C_{i, j, k}^{n+1}}{\Delta y^{2}}-\mu_{y} \frac{C_{i, j, k}^{n+1}-C_{i, j-1, k}^{n+1}}{\Delta y^{2}}= \\
& =M_{y y}^{-} C^{n+1}+M_{y y}^{+} C^{n+1}, \\
& \frac{\partial}{\partial z}\left(\mu_{z} \frac{\partial C}{\partial z}\right) \approx \mu_{z} \frac{C_{i, j, k+1}^{n+1}-C_{i, j, k}^{n+1}}{\Delta z^{2}}-\mu_{z} \frac{C_{i, j, k}^{n+1}-C_{i, j, k-1}^{n+1}}{\Delta z^{2}}= \\
& =M_{z z}^{-} C^{n+1}+M_{z z}^{+} C^{n+1}, \\
& \frac{\partial u^{+} C}{\partial x} \approx \frac{u_{i+1, j, k}^{+} C_{i, j, k}^{n+1}-u_{i, j, k}^{+} C_{i-1, j, k}^{n+1}}{\Delta x}=L_{x}^{+} C^{n+1}, \\
& \frac{\partial u^{-} C}{\partial x} \approx \frac{u_{i+1, j, k}^{-} C_{i+1, j, k}^{n+1}-u_{i, j, k}^{-} C_{i, j, k}^{n+1}}{\Delta x}=L_{x}^{-} C^{n+1}, \\
& \frac{\partial v^{+} C}{\partial y} \approx \frac{v_{i, j+1, k}^{+} C_{i, j, k}-v_{i, j, k}^{+} C_{i, j-1, k}}{\Delta y}=L_{y}^{+} C^{n+1}, \\
& \frac{\partial v^{-} C}{\partial y} \approx \frac{v_{i, j+1, k}^{-} C_{i, j+1, k}-v_{i, j, k}^{-} C_{i, j, k}}{\Delta y}=L_{y}^{-} C^{n+1}, \\
& \frac{\partial w^{+} C}{\partial z} \approx \frac{w_{i, j, k+1}^{+} C_{i, j, k}-w_{i, j, k}^{+} C_{i, j, k-1}}{\Delta z}=L_{z}^{+} C^{n+1}, \\
& \frac{\partial w^{-} C}{\partial z} \approx \frac{w_{i, j, k+1}^{-} C_{i, j, k+1}-w_{i, j, k}^{-} C_{i, j, k}}{\Delta z}=L_{z}^{-} C^{n+1} .
\end{aligned}
$$

The computational dependencies for the first equation from (6) are [12]:

- on the first step of splitting $k=n+1 / 4$ :

$$
\frac{C_{i, j, k}^{k}-C_{i, j, k}^{n}}{\Delta t}+\frac{1}{2}\left(L_{x}^{+} C^{k}+L_{y}^{+} C^{k}+L_{z}^{+} C^{k}\right)=0
$$

- on the second step of splitting $\mathrm{k}=\mathrm{n}+1 / 2, \mathrm{c}=\mathrm{n}+1 / 2$ :

$$
\frac{C_{i, j, k}^{k}-C_{i, j, k}^{c}}{\Delta t}+\frac{1}{2}\left(L_{x}^{-} C^{k}+L_{y}^{-} C^{k}+L_{z}^{-} C^{k}\right)=0
$$


- on the third step of splitting $\mathrm{k}=\mathrm{n}+3 / 4, \mathrm{c}=\mathrm{n}+1 / 2$ :

$$
\frac{C_{i, j, k}^{k}-C_{i, j, k}^{c}}{\Delta t}+\frac{1}{2}\left(L_{x}^{-} C^{k}+L_{y}^{-} C^{k}+L_{z}^{-} C^{k}\right)=0
$$

- on the fourth step of splitting $k=n+1 ; c=n+3 / 4$ :

$$
\frac{C_{i, j, k}^{k}-C_{i, j, k}^{n}}{\Delta t}+\frac{1}{2}\left(L_{x}^{+} C^{k}+L_{y}^{+} C^{k}+L_{z}^{+} C^{k}\right)=0
$$

Considered splitting scheme is called alternately triangular. On the bases of dependencies (8), (9) concentration of hazardous substances in the difference cells under the influence of air flow is computed using «running calculation».

Next calculation of concentration changing in cells under the influence of diffusion is made:

- on the first step of splitting [11]:

$$
\begin{gathered}
\frac{C_{i, j, k}^{n+\frac{1}{2}}-C_{i, j, k}^{n}}{\Delta t}=\left[\mu_{x} \frac{-C_{i, j, k}^{n+\frac{1}{2}}+C_{i-1, j, k}^{n+\frac{1}{2}}}{\Delta x^{2}}\right]+ \\
+\left[\mu_{y} \frac{-C_{i, j, k}^{n+\frac{1}{2}}+C_{i, j-1, k}^{n+\frac{1}{2}}}{\Delta y^{2}}\right]+\left[\mu_{z} \frac{-C_{i, j, k}^{n+\frac{1}{2}}+C_{i, j, k-1}^{n+\frac{1}{2}}}{\Delta z^{2}}\right]
\end{gathered}
$$

- on the second step of splitting [11]:

$$
\begin{gathered}
\frac{C_{i, j, k}^{n+1}-C_{i, j, k}^{n+\frac{1}{2}}}{\Delta t}=\left[\mu_{x} \frac{C_{i+1, j, k}^{n+1}-C_{i, j, k}^{n+1}}{\Delta x^{2}}\right]+ \\
+\left[\mu_{y} \frac{C_{i, j+1, k}^{n+1}-C_{i, j, k}^{n+1}}{\Delta y^{2}}\right]+\left[\mu_{z} \frac{C_{i, j, k+1}^{n+1}-C_{i, j, k}^{n+1}}{\Delta z^{2}}\right]
\end{gathered}
$$

On the bases of dependencies (10), (11) concentration of hazardous substances in the difference cells is computed using «running calculation».

The final stage is calculating of hazardous substance concentration under the action of emission sources and precipitations. So, for solving of the third equation from system (6) dependency (12) is used (obtained on the base of Euler method [11]):

$$
\begin{gathered}
C_{i j k}^{n+1}=C_{i j k}^{n+A}+ \\
+\Delta t \sum Q_{i j k}(t) \delta_{l}\left(x-x_{i}(t)\right) \delta_{l}\left(y-y_{i}(t)\right) \delta_{l}\left(z-z_{i}\right) .
\end{gathered}
$$

Here the following designation of hazardous substance emission rate is used:

$$
Q_{i j k}=Q_{k} / \Delta x / \Delta y / \Delta z
$$

where $Q_{k}$ is known emission rate value of source.

To solve equation (7) dependency (13), obtained from Euler method, is used:

$$
C_{i j k}{ }^{n+1}=C_{i j k}{ }^{n}-\Delta t \sigma C_{i j k}{ }^{n}
$$

Initial condition for each differential equation is written as following:

$$
C^{1}\left|=C\left(x, y, t^{n}\right) ; C^{k}\right|=C^{k-1} \mid .
$$

\section{Methodology of territorial risk assessment}

As known, spreading of dangerous factor in case of toxic substances accidental emission has significantly probabilistic nature. This nature of spreading is depended from:

- the repetition of different velocity values, the direction of the wind;

- probability of atmospheric stability;

- accident scenario;

- place of emission, emission rate.

Therefore, in assessing the territorial risk, we will take into account these factors. Note that the zone of chemical contamination is different for different meteorological situations $P\left(W_{i}\right)$.

Indeed, at high air velocity the concentration of dangerous substance in the air decreases, and at low speed it increases, because there is no rapid dispersion. In addition, the process of dispersion of contaminations is influenced by the state of the atmosphere, which in the constructed models is taken into account by the vertical coefficient of atmospheric diffusion.

When constructing a method of risk assessment, by a specific meteorological situation the specific significance of the velocity and direction of the wind and the stability of the atmosphere is meant. As known, the probability of realization of a concrete meteorological situation for a given region is determined on the basis of statistical data (or on the basis of meteorological forecasting):

$$
P\left(W_{i}\right)=N_{\Pi} / \mathrm{T},
$$

where $N_{\Pi}$ is number of days (hours), corresponding to a particular meteorological situation; $T$ is period of observing.

The probability of a person is in the zone of defeat will be determined as follows:

$$
P(W)_{\Sigma}=\sum_{i=0}^{n} P\left(W_{i}\right)
$$

where $P(W)_{\Sigma}$ is probability of all considered meteorological situations, in which a person is in a zone where the concentration of a dangerous substance exceeds a certain threshold, at which there is a damage of certain severity.

To calculate the probability of being in a zone of toxic damage when emitting a dangerous substance it is necessary, for a specific point of the calculated area, to perform calculations by the formula (15). 


\section{Algorithm of solving}

The territorial risk assessment of chemical damage in the probable meteorological situation $P(W)$ will be carried out in the following sequence:

1) at the first stage of the task solving, a data block is created to initiate the event (possible place of emission of a chemically hazardous substance, emission rate, emission mode, type of chemical agent);

2) at the second stage, a data block is formed about probable meteorological conditions $P\left(W_{i}\right)$, characteristic of the area where the railway is located;

3 ) at the third stage, the level of chemical contamination for probable meteorological conditions is calculated (at this stage the numerical integration of the masstransfer equation for a particular meteorological situation is carried out);

4) at the fourth stage, the zones where the concentration exceeds the threshold (for example, lethal concentration, Maximum permissible concentration etc.) in a specific meteorological situation are determined;

5) at the fifth stage, the construction of a matrix (risk matrix) for the given object is carried out.

To perform calculations in order to determine the value of the territorial risk of chemical damage in the case of an accident on the railway, the following parameters should be specified:

1. Dimensions of calculated zone.

2. Location of railway.

3. Location of inhabited area.

4. Meteorological parameters.

5. Hazardous substances emission rate.

6. Emission source coordinates.

7. Receptor coordinates.

8. Probability of each meteorological situation.

The result of the simulation is the matrix of the territorial risk or isoline of the concentration of the toxic substance. If necessary, the user can "include" in the calculation the probability of an accident location, and so on. Based on the developed algorithm, a computer program «PR-Prob» was created. FORTRAN was used for coding difference equations.

\section{Results}

The developed «PR-Prob» was used to solve the following problem. A train with liquid ammonia $\left(\mathrm{NH}_{3}\right)$ moves near Railway Station «Kamyanske» (Dnipropetrovsk Region, Ukraine) and at time $\mathrm{t}=0$ the instant emission of ammonia takes place. It is assumed that at time $\mathrm{t}=0$, the amount of emitted $\mathrm{NH}_{3}$ was $\mathrm{Q}=6$ ton (it means $10 \%$ of the total amount of liquid cargo). Because of this emission, toxic chemical cloud is formed near the railway (Figure 1, dimensions of the computational region: $1.75 \mathrm{~km} \times 1.3 \mathrm{~km})$.

The train keeps moving after the toxic chemical emission and long term emission of $\mathrm{NH} 3(\mathrm{Q}=10 \mathrm{~kg} / \mathrm{s})$ follows the instant emission. Therefore, there is following scenario of complex emission: «instant exhaust of toxic chemical + long term emission of it».
Formation of polluted zones in the atmosphere after the accident is shown in Figures 2, 3. Computation results were obtained for wind speed $7 \mathrm{~m} / \mathrm{s}$, train speed $8 \mathrm{~m} / \mathrm{s}$.

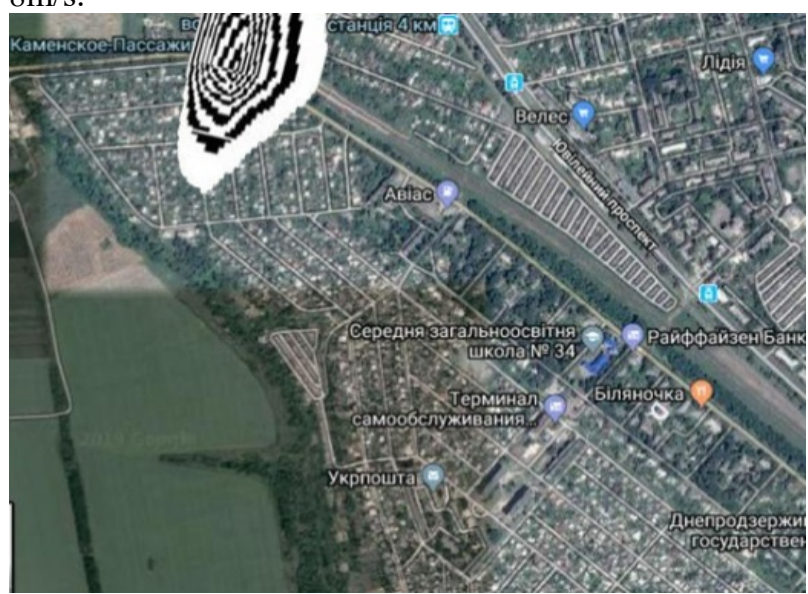

Fig. 1. Toxic chemical cloud after $\mathrm{NH}_{3}$ emission, $t=3.5 \mathrm{~min}$, level $z=7 \mathrm{~m}$

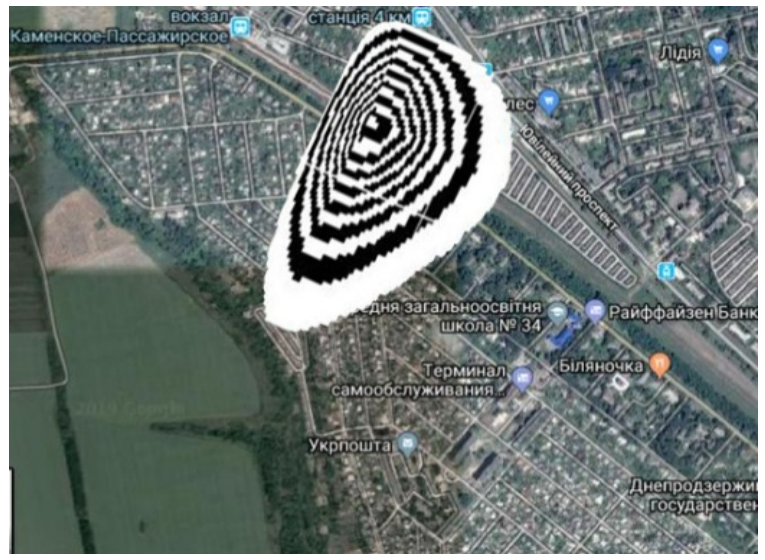

Fig. 2. Toxic chemical cloud after $\mathrm{NH}_{3}$ emission, $t=12 \mathrm{~min}$, level $z=7 \mathrm{~m}$

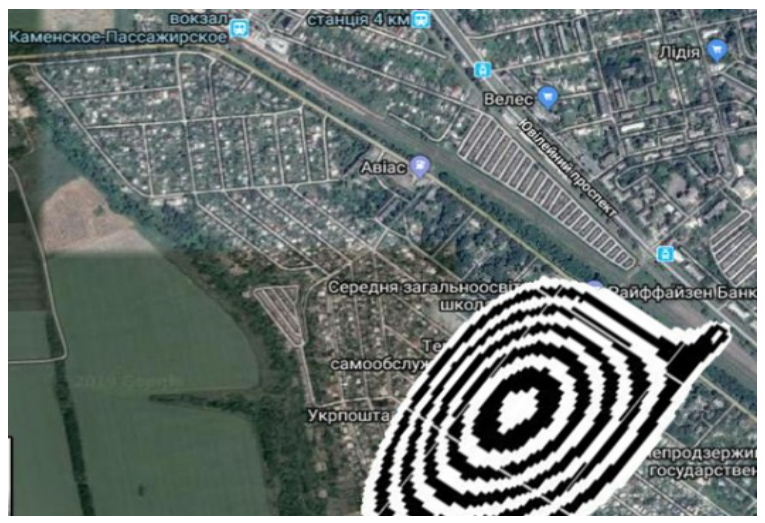

Fig. 3. Toxic chemical cloud after $\mathrm{NH}_{3}$ emission, $t=18 \mathrm{~min}$, level $z=7 \mathrm{~m}$

It is clear from Figures 2, 3 that in the case of accident large cloud of pollution is formed near the railway with further covering the inhabited area and creating the threat of toxic damage for people.

In Figure 4 the matrix of potential territorial risk for the meteorological situations, which are typical for this region in June, is formed. 


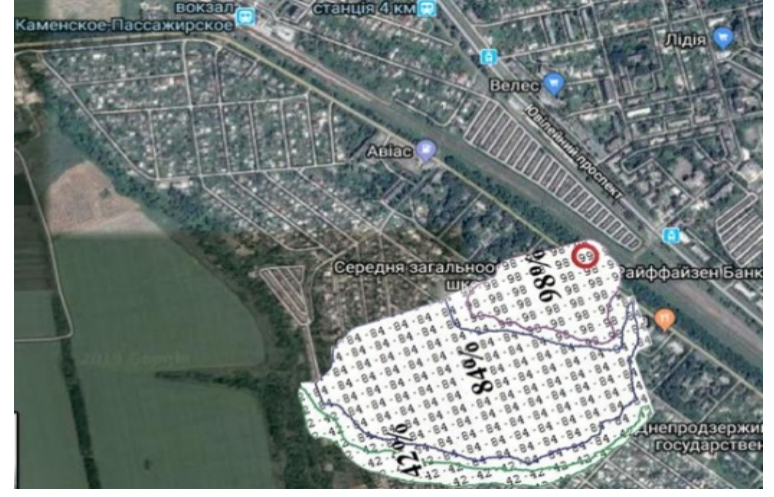

Fig. 4. Matrix of potential territorial risk, $t=15 \mathrm{~min}$, level $z=2 \mathrm{~m}$

Matrix of potential territorial risk shows the probability of people toxic damage at any time after the accident. This information allows us to separate zones, which are extremely dangerous for people in inhabited area for all possible weather conditions in the region.

In Table 1 we present computational data about the dynamics of land surface contamination. As we see from Table 1, the contaminated surface is increased during time.

Table 1. Dynamics of land surface contamination.

\begin{tabular}{|c|c|c|c|}
\hline Time, $\mathrm{s}$ & 112 & 169 & 239 \\
\hline $\begin{array}{c}\text { Contaminated } \\
\text { area, } \mathrm{m}^{2}\end{array}$ & $0,21 \times 10^{6}$ & $1,04 \times 10^{6}$ & $1,86 \times 10^{6}$ \\
\hline
\end{tabular}

In Table 2 we present computational data about the dynamics pollutant mass settled on the land after the accident. This data allows to assess the damage after the accident and can be used to calculate economic losses, caused by the accident. So, in case of accidents during transportation, the developed model allows to make platform of scientific justification losses, caused by an accident.

Table 2. Pollutant mass settled on the land, g.

\begin{tabular}{|c|c|}
\hline Time, $\min$ & Area \\
\hline 4 & $0,11 \times 10^{6}$ \\
\hline 14 & $0,27 \times 10^{6}$ \\
\hline 22 & $0,38 \times 10^{6}$ \\
\hline
\end{tabular}

\section{Conclusions}

The paper shows the numerical approach to risk assessment in case of toxic chemicals emissions during railway transportations. This approach is based on numerical simulation of toxic chemical dispersion on the base of $3 \mathrm{D}$ equation of mass transport. For numerical integration of the governing equation, implicit difference schemes of splitting are used. The unknown value of toxic chemical concentration is calculated using explicit formulae on each step of splitting.

The proposed numerical models can be used to compute zones near railways which are influenced by the emissions from railway transport.

\section{References}

1. V.A. Kotlyarevskii, A.V. Zabegayeva, Accidents and disasters: caution and aftermath liquidation, (ASV, Moscow, 2001).

2. M.M. Biliaiev, E.Yu. Gunko, P.B. Mashykhina, Mathematical modeling in tasks of ecological safety and emergencies monitoring, (Akcent PP, Dnipro, 2013).

3. M.M. Biliaiev, O.V. Berlov, Cal. of sc. works NGU, 42, 160-167, 2013.

4. O.V. Berlov, Sc. bul. of civ. eng., 1(75), 185-189, 2014.

5. M.E. Berlyand, Modern problems of atmosphere diffusion and atmosphere pollution, (Gidrometeoizdat, Leninhrad, 1975).

6. N.G. Gusiev, V.A. Belyaev, Radioactive releases in biosphere, (Energoatomizdat, Moscow, 1991).

7. Yu.A. Izrael, Ecology and monitoring the environment state, (Gidrometeoizdat, Moscow, 1984).

8. G.I. Marchuk. Mathematical modeling in environmental problems, (Nauka, Moscow, 1982).

9. P.B. Mashykhina, Bul. of Dn. nat. univ. of rai. tr., 27, 138-142, 2009.

10. T.I. Rusakova, Bul. of Dn. nat. univ. of rai. tr., 6 (48), 32-45, 2013.

11. A.A. Samarsky, Theory of difference schemes, (Nauka, Moscow, 1983).

12. M.Z. Zgurovsky, V.V. Skopetsky, V.K. Khrusch, M.M. Biliaiev, Numerical modeling of pollution spreading in environment, (Nauk. dumka, Kyiv, 1997).

13. A.A. Shvyriaev, V.V. Menshykov, Risk assessment of the impact of pollution in investigated region, (MGU, Moscow, 2004).

14. M.M. Biliaiev, NATO Science for Peace and Security. Series C: Environmental Security, 87-91, 2012.

15. Michal Kisa, Ludovit Jelemensky and Jan Stopka, IChemE Symposium series № 153, 1-8, 2007.

16. Ondrej Zavila, Pavel Dobes, Jakub Dlavka, Jan Bitta, The science for population protection, 2, 1-8, 2015.

17. Principles for environmental risk assessment of the sediment compartment: Proceedings of the topical scientific workshop, (European Chemicals Agency, Helsinki, Finland, 2014).

18. Risk Assessment Methodology for Hazardous Substances. How to assess the risk, cost and benefit of new hazardous substances for use in New Zealand, (New Zealand, 2018).

19. Risk Assessment for Toxic Air Pollutants: A Citizen's Guide, (EPA, USA, 1991).

20. Werner K. Graber, Fritz Gassmann, Mathematics and Computers in Simulation (MATCOM), Paul Scherrer Institute, 52, 413-426, 2000. 